OPEN ACCESS

Edited by:

Kuirong Jiang,

Nanjing Medical University, China

Reviewed by:

Xi Yang,

Fudan University, China

Pierpaolo Correale,

Azienda ospedaliera 'Bianchi-

Melacrino-Morelli', Italy

*Correspondence:

Juan Du

dujuanglyy@163.com

Baorui Liu

baoruiliu@nju.edu.cn

${ }^{+}$These authors have contributed equally to this work

Specialty section:

This article was submitted to Gastrointestinal Cancers,

a section of the journal

Frontiers in Oncology

Received: 10 April 2021 Accepted: 23 September 2021 Published: 13 October 2021

Citation:

Dai W, Qiu X, Lu C, Zou Z, Sha H, Kong W, Liu B and Du J (2021) AGIG Chemo-Immunotherapy

in Patients With Advanced

Pancreatic Cancer: A Single-Arm, Single-Center, Phase 2 Study.

Front. Oncol. 11:693386. doi: 10.3389/fonc.2021.693386

\section{AGIG Chemo-Immunotherapy in Patients With Advanced Pancreatic Cancer: A Single-Arm, Single-Center, Phase 2 Study}

\author{
Wangshu Dai ${ }^{1,2 \dagger}$, Xin Qiu ${ }^{3 \dagger}$, Changchang $\mathrm{Lu}^{3 \dagger}$, Zhengyun Zou ${ }^{1}$, Huizi Sha ${ }^{1}$, \\ Weiwei Kong ${ }^{1}$, Baorui Liu ${ }^{1 *}$ and Juan Du ${ }^{1,3^{*}}$
}

1 The Comprehensive Cancer Center of Drum Tower Hospital, Medical School of Nanjing University \& Clinical Cancer Institute of Nanjing University, Nanjing, China, ${ }^{2}$ The Cadre Health Care Ward, Nanjing Drum Tower Hospital, The Affiliated Hospital of Naniing University Medical School, Naniing, China, ${ }^{3}$ The Comprehensive Cancer Center of Drum Tower Hospital, Clinical College of Traditional Chinese and Western Medicine, Nanjing University of Chinese Medicine, Nanjing, China

Background: To date, chemotherapy remains the only effective treatment of unresectable pancreatic adenocarcinoma. In the past few years, the interest in immunological anticancer therapy rises sharply. AGIG is a novel chemoimmunotherapy regimen that combines nab-paclitaxel + gemcitabine chemotherapy with sequential recombinant interleukin-2 (IL-2) and granulocyte-macrophage colony stimulating factor (GM-CSF) therapy. We conducted a single-arm prospective phase II study to determine the efficacy and safety of the first-line treatment of advanced pancreatic cancer with $A G I G$ regimen.

Methods: Nab-paclitaxel $\left(125 \mathrm{mg} / \mathrm{m}^{2}\right)$ and gemcitabine $\left(1000 \mathrm{mg} / \mathrm{m}^{2}\right)$ were administered intravenously to all patients on days 1 and 8 triweekly, interleukin-2 (1000000U) and GMCSF $(100 \mu \mathrm{g})$ were administered subcutaneously on days 3-5 after chemotherapy. The primary end point was ORR by the Response Evaluation Criteria in Solid Tumors, version 1.1. Secondary end points included safety profile, progression-free survival (PFS), overall survival (OS). Patients' conditions along with the efficacy and safety were assessed every two cycles.

Results: Between 11/2018 and 01/2020, sixty-four patients were enrolled. In the sixtyfour evaluable patients, the disease control rate (DCR) and overall response rate (ORR) were $76.6 \%$ and $43.75 \%$, respectively. The median follow-up time was 12.1 (range $7.1-$ 22.4) months. The median PFS was 5.7 (range 1.63-15.8) months. The median OS was 14.2 (range 2.9-22.0) months. The most common adverse event was fever (75\%). The incidence of III/IV grade neutropenia was $4.69 \%$. In subgroup analyses, we found that 
eosinophil count in the blood elevated three times higher than baseline level predicted a longer survival.

Conclusions: The AGIG chemo-immunotherapy regimen has presented favorable ORR, OS, and manageable toxicities as first-line therapeutic strategy of advanced pancreatic cancer treatment. This regimen may be a novel reliable therapeutic option for patients with preserved performance status. The improvement of treatment efficiency may be related to the activation of non-specific immune response.

\section{Clinical Trial Registration: https://clinicaltrials.gov/. identifier NCT03768687.}

Keywords: objective response rate, overall survival, advanced pancreatic adenocarcinoma, chemoimmunotherapy, nab-paclitaxel, gemcitabine

\section{INTRODUCTION}

Pancreatic cancer is one of the deadliest solid malignancies in the world. Despite decades of efforts, it remains the fourth leading cause of cancer-related death worldwide, with a five-year survival rate of less than $5 \%$ (1). Without treatment, the median survival time is consistently shorter than six months (2). Since 1997, gemcitabine had been the standard treatment for unresectable pancreatic adenocarcinoma (3). After decades of exploration, both FOLFIRINOX (fluorouracil, irinotecan, and oxaliplatin) and nab-paclitaxel with gemcitabine (AG) prolong overall survival (OS) compared with gemcitabine alone $(4,5)$. Till now, chemotherapy remains the only effective treatment of unresectable pancreatic adenocarcinoma (6).

As mentioned above, FOLFIRINOX is one of the standard treatment strategies for patients with advanced pancreatic cancer and has demonstrated good effectiveness in Europe and North America $(7,8)$. However, grade III/IV adverse events were commonly observed in the FOLFIRINOX treatment courses. To our knowledge, cancer drug tolerability is different between Asian and white populations. These differences may be related to genetic or environmental factor. Increased chemotherapy-induced myelosuppression was one of the most commonly observed adverse events in Asian patients $(9,10)$. Chinese patients were unendurable to FOLFIRINOX chemotherapy sometimes. The combination of nabpaclitaxel and gemcitabine is recommended as the first-line treatment regimen for patients with advanced pancreatic cancer by the National Comprehensive Cancer Network (NCCN) guidelines. A phase I/II study evaluated the AG chemotherapy regimen in Chinese patients with advanced pancreatic cancer (11). The study was carried out at a dose and schedule different from the classic MPACT study. The recommended administration schedule was described as follows, nab-paclitaxel $\left(125 \mathrm{mg} / \mathrm{m}^{2}\right)$ along with gemcitabine $\left(1000 \mathrm{mg} / \mathrm{m}^{2}\right)$ was administered on the first day and the eighth day, the treatment was repeated every three weeks (12). Although the trail did not meet its primary endpoint of identifying the maximum tolerated dose in Chinese pancreatic cancer sufferers, the study showed a manageable safety profile with a favorable antitumor effect in pancreatic cancer sufferers.

With the clinical development and application of PD-1/PD-L1 immune-checkpoint blockade, the interest in the exploration of immunological anticancer strategies rises sharply in these years.
Immune-based regimens are showing promise where other approaches have failed when treating pancreatic cancer $(13,14)$. Immune checkpoint inhibitors along with therapeutic vaccines and combination immunotherapies are commonly used as immunotherapeutic strategies. Even though the antitumor effect and mechanism of the above-mentioned immunotherapeutic strategies remain unclear, these researches produced abundant data concerning the mechanisms of the efficient tumor-specific adaptive immune response triggered by immune-modulating agents (15).

It was reported that chemo-immunotherapy might represent as an innovative reliable therapy option for first-line treatment of metastatic colorectal cancer sufferers $(16,17)$. Interleukin-2 (IL-2) was used to promote the proliferation of cross-primed cytotoxic $\mathrm{T}$ lymphocyte clones, while granulocyte-macrophage colony stimulating factor (GM-CSF) was required to activate the antigen-presenting ability of the dendritic cells expressed in human peripheral blood mononuclear cells. GM-CSF is essential for the differentiation of dendritic cells, which are responsible for processing and presenting tumor antigens for the priming of antitumor cytotoxic T lymphocytes $(18,19)$. Some GM-CSF-based cancer immunotherapy strategies have been developed for in clinical practice (20). It was reported that IL-2 and GM-CSF were demonstrated as innovative and reliable adjuvants of chemotherapy for metastatic colorectal cancer $(21,22)$. These results offered the rationale to design a novel treatment chemo-immunotherapy regimen that combines traditional chemotherapy with IL-2 and GM-CSF.

AGIG is a novel chemo-immunotherapy regimen that combines AG chemotherapy with sequential recombinant IL-2 and GM-CSF therapy (nab-paclitaxel, gemcitabine, IL-2 and GM-CSF). In this study, we implemented a single-arm, singlecenter prospective phase II study to determine the efficacy and safety of the AGIG regimen as the first-line treatment of advanced pancreatic cancer in China.

\section{MATERIALS AND METHOD}

\section{Patients}

This was a prospective study involving pancreatic sufferers receiving AGIG Chemo-immunotherapy regimen from 
November 2018 to January 2020 at the Comprehensive Cancer Centre of Drum Tower Hospital, Clinical Cancer Institute of Nanjing University. In all cases, a multidisciplinary team participated in the diagnosis of pancreatic adenocarcinoma followed by the NCCN guidelines. Patients with Eastern Cooperative Oncology Group (ECOG) performance score higher than 1, inadequate bone marrow, abnormal liver or renal functions, additional other malignancies, and patients older than eighty-five years were excluded. Patients enrolled in the trial were prescribed AGIG regimen.

\section{Procedures}

Patients enrolled in the trial received AGIG chemoimmunotherapy. Nab-paclitaxel $(125 \mathrm{mg} / \mathrm{m} 2)$ and gemcitabine $(1000 \mathrm{mg} / \mathrm{m} 2)$ were administered intravenously to all patients on the first day and the eighth day of the treatment cycle. IL-2 $(10000000 \mathrm{U})$ and GM-CSF $(100 \mu \mathrm{g})$ were administered subcutaneously on three to five days after chemotherapy. The treatment is repeated every three weeks. Figure 1 showed the drug administration protocol of the AGIG regimen. We evaluate clinical and laboratory results at baseline and repeated every time before chemotherapy. Radiographic response evaluation was performed every six weeks. Subjects continued their treatment until disease progression, clinical judgment, occurrence of unacceptable toxicity, or withdrawal of consent. Supportive care was permitted during the treatment course. Second line therapy after disease progression was left to the discretion of the treating oncologist.

\section{Assessment}

All patients were evaluated every two cycles of AGIG chemoimmunotherapy using multislice computed tomography scans with contrast medium. Physical examination and laboratory tests including blood routine test, biochemical index and serum CA199 assays were performed every time before chemotherapy. We categorize tumor response into complete response (CR), partial response (PR), stable disease (SD), and progressive disease (PD) according to the Response Evaluation Criteria in Solid Tumors (version 1.1). With respect to the safety observation of the treatment, we graded adverse events according to the National Cancer Institute Common Terminology Criteria for Adverse Events (version 4.0). Overall survival (OS) was defined as the duration from the beginning of chemotherapy to the date of death of any cause. Progression free survival (PFS) was defined as the duration from the beginning of chemotherapy to the date of disease progression or death. Subjects without event were censored at the last follow-up date (August 1st, 2020). Characteristic files were collected at the moment of admission.

\section{Statistical Analysis}

All data were analyzed using Graphpad Prism 6 and SPSS software (version 21.0). Survival analyses were performed using the Kaplane-Meier method, Log-rank (Mantel-Cox) tests and Gehan-Breslow-Wilcoxon tests. Data were presented as median and range. A $\mathrm{P}$ value less than 0.05 was considered statistical significance.

\section{RESULTS}

\section{Patient Characteristics}

Between 11/2018 and 01/2020, a total of sixty-four patients were enrolled and evaluated in our trial. Figure 2 showed the study flowchart of this trial. Patient characteristic files at baseline are summarized in Table 1. There were thirty-six (56.25\%) males and twenty-eight (43.75\%) females. The median age was 62 (range 33 81 ) years. All subjects were ECOG PS 0-1. Fifty-nine PC sufferers (92.19\%) had elevated baseline CA199, with a median value of 1033 (range 27 - 30491) u/mL. In total, 51.56\% ( $n=33$ ) of the tumors were located in the head and neck of the pancreas. $40.63 \%$ $(n=26)$ of the tumors were located in the body or tail of the pancreas. 49 patients were histologically diagnosed as adenocarcinoma including one case of cystadenocarcinoma and one case of mucinous adenocarcinoma. 4 patients were histologically diagnosed as adeno-squamous carcinoma, and none of the patients developed undifferentiated or undifferentiated neuroendocrine carcinoma. In addition, 11 patients' pathological types are still unknown due to the limited pathologic sampling ability of endoscopic ultrasonography. Nearly half of enrolled patients ( $\mathrm{n}=31,48.44 \%)$ had metastases at the initial diagnosis. The majority of the cases had liver $(n=23,74.19 \%)$ or peritoneal $(\mathrm{n}=10,32.26 \%)$ metastasis. Seven patients had undergone a prior resection.

\section{Treatment Completion Rates}

Seventy-four subjects were assessed for eligibility initially. Six patients were excluded because they did not meet inclusion criteria. Four patients had abnormal baseline laboratory results and two patients had immeasurable disease. Sixty-eight patients were allocated to intervention. Four patients did not receive allocated intervention, two for patient preference and two for unknown reason. A total of sixty-four patients proceeded to AGIG and were analyzed for toxic effects and efficacy. Forty-eight patients were observed disease progression throughout the follow up. Seven patients received surgical resection. Seven patients had been receiving maintenance therapy till the last follow-up date (August 1st, 2020). Two patients drop out the trial, 1 with obstructive jaundice and 1 with femoral head necrosis.

\section{Radiographic Response Evaluation}

Radiographic response was measured with RECIST 1.1 every two cycles of AGIG chemo-immunotherapy. With sixty-four patients evaluated, two patients discontinued chemotherapy early because of obstructive jaundice and femoral head necrosis. Twenty-eight patients (43.75\%) had PR, twenty-one patients (32.81\%) had SD, and fifteen patients (23.43\%) had PD. Table 2 summarized the detail information of best response. For all patients $(n=64)$, the overall response rate $($ ORR $)$ and disease control rate (DCR) was $43.75 \%$ and $76.6 \%$ respectively. No significant difference in treatment response rate was observed between the two primary tumor sites.

\section{Survival Analysis and Subgroup Analysis}

At the last follow-up (1st August 2020), thirty-two patients (50\%) had died. All sixty-four patients were included for 
TABLE 1 | Baseline characteristics. (intention-to-treat population).

\begin{tabular}{|c|c|c|}
\hline Characteristic & AGIG (N = 64) & No. (\%) \\
\hline \multicolumn{3}{|l|}{ Sex } \\
\hline Male & 36 & $56.25 \%$ \\
\hline Female & 28 & $43.75 \%$ \\
\hline Age, median (range),y & $62(33-81)$ & \\
\hline \multicolumn{3}{|l|}{ ECOG PS } \\
\hline 0 & 19 & $29.70 \%$ \\
\hline 1 & 45 & $70.30 \%$ \\
\hline \multicolumn{3}{|l|}{ CA 19-9 level at baseline } \\
\hline median (range), u/ml & $1033(27-30491)$ & \\
\hline$<37 \times$ ULN Normal & 28 & $43.75 \%$ \\
\hline$\geq 37 \times$ ULN Normal & 31 & $48.43 \%$ \\
\hline Unknown & 5 & $7.80 \%$ \\
\hline \multicolumn{3}{|l|}{ Tumor site } \\
\hline Head and neck & 33 & $51.56 \%$ \\
\hline Body and tail & 26 & $40.63 \%$ \\
\hline Unknown & 5 & $7.81 \%$ \\
\hline \multicolumn{3}{|l|}{ Histology } \\
\hline Adenocarcinoma & 49 & $76.56 \%$ \\
\hline Adeno-squamous carcinoma & 4 & $6.25 \%$ \\
\hline Unclear & 11 & $17.19 \%$ \\
\hline \multicolumn{3}{|l|}{ Stage } \\
\hline Resection & 7 & $10.94 \%$ \\
\hline locally advanced & 26 & $40.63 \%$ \\
\hline Metastatic & 31 & $48.44 \%$ \\
\hline Site of metastatic disease & $N=31$ & No.(\%) \\
\hline Liver & 23 & $74.19 \%$ \\
\hline Lung & 4 & $12.90 \%$ \\
\hline Peritoneum & 10 & $32.26 \%$ \\
\hline Bone & 2 & $6.45 \%$ \\
\hline \multicolumn{3}{|l|}{ No. of metastatic sites } \\
\hline 1 & 1 & $3.23 \%$ \\
\hline 2 & 5 & $16.13 \%$ \\
\hline 3 & 1 & $3.23 \%$ \\
\hline$>3$ & 24 & $77.25 \%$ \\
\hline
\end{tabular}

survival analysis. The median follow-up time was 12.1 (range 7.1-22.4) months. For all patients, the median PFS was 5.7 (range 1.63-15.8) months (Figure 3A), the median OS was 14.2 (range 2.9-22.0) months (Figure 3B), and the one-year survival rate was $65 \%$. We performed subgroup survival analyses in CA199 level (Figure 4A), eosinophil count variation (Figure 4B), NK cell count variation (Figure 4C) and $\mathrm{CD} 3+\mathrm{CD} 4 / \mathrm{CD} 3+\mathrm{CD} 8+$ proportion (Figure 4D). We found that eosinophil count in the blood elevated three times higher than baseline level predicted a longer survival $(\mathrm{P}=0.016)$ (Figure 4B).

\section{Adverse Events}

The therapy-related toxicities are summarized in Table 3. Generally, the incidence of adverse events was $79.69 \%(n=51)$, and fever was the most common side effect, with an incidence of $75 \%$. For severe adverse events, thirty-eight patients $(59.38 \%$ ) were observed with grade III/IV toxicities, among which $84.21 \%$ $(\mathrm{n}=32)$ was alopecia, 26.32\% $(\mathrm{n}=10)$ was peripheral sensory neuropathy, $7.89 \%(\mathrm{n}=3)$ was neutropenia, $13.16 \%(\mathrm{n}=5)$ was thrombocytopenia, $10.53 \%(\mathrm{n}=4)$ was anemia. Totally, three patients $(4.69 \%)$ received granulocyte-colony stimulating factor treatment before or after chemotherapy. In addition, five patients
(3.2\%) were treated with thrombopoietin. No patients suffered adverse event leading to death.

\section{DISCUSSION}

Generally, advanced pancreatic ductal adenocarcinoma is considered an incurable presentation of PC. This trial was carried out to investigate the efficacy and safety of the AGIG regimen in Chinese patients with advanced PC. In the present study, the ORR was $43.75 \%$, with a significantly higher ORR compared with MPACT study $(22.96 \%, \mathrm{n}=431)(\mathrm{p}=0.0007)(12)$ and a slightly higher ORR compared with LAPACT study (33.64\%, n=107) (23) and HALO 202 study (32.60\% n=92) (3). Other efficacy endpoints (PFS, 5.7 months; OS, 14.2 months) were not inferior to the findings of the MPACT study (PFS, 5.5 months; OS, 8.5 months) (12) and the study of Karasic et al. (PFS, 6.4 months; OS, 12.1 months) (24). The PFS was a little shorter in this study than that in the study of Karasic et al. (5.7 months vs 5.5 months). We hypothesize that these discrepancies are attributed to differences in the radiographic response measuring frequency, with biweekly measurement in this study versus triweekly measurement in the study of Karasic et al. (24). 


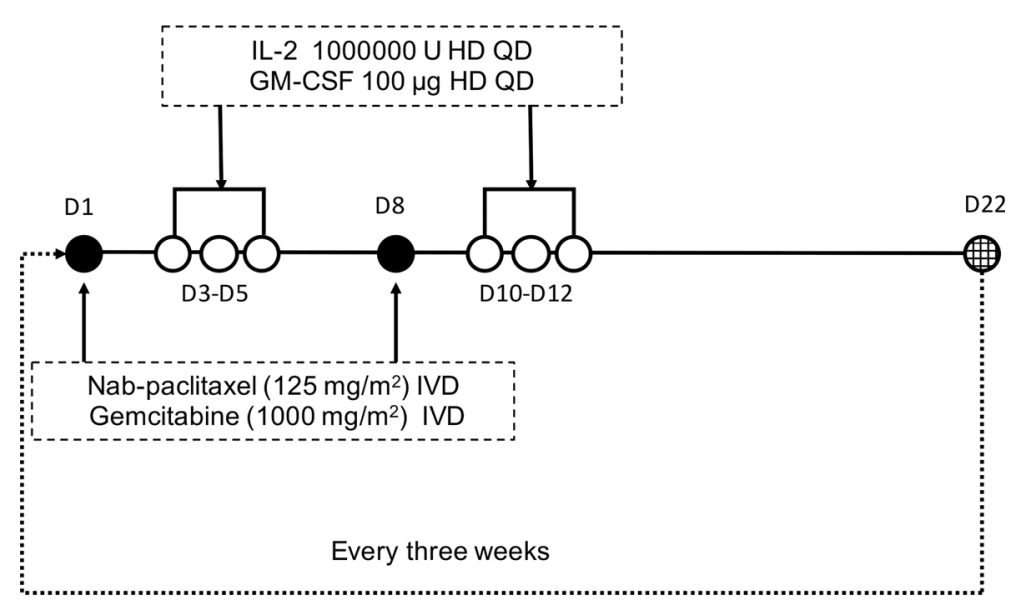

FIGURE 1 | Protocol of drug administration. Nab-paclitaxel $\left(125 \mathrm{mg} / \mathrm{m}^{2}\right)$ and gemcitabine $\left(1000 \mathrm{mg} / \mathrm{m}^{2}\right)$ were administered intravenously to all patients on days 1 and 8 triweekly. Interleukin-2 (10000000 U) and GM-CSF (100 $\mu \mathrm{g})$ were administered subcutaneously on days 3-5 after chemotherapy.

74 Patients assessed for eligibility

6 Excluded because did not meet inclusion criteria 4 Abnormal baseline laboratory results

2 Unmeasurable disease

68 Allocated to intervention

64 Received allocated intervention

4 Did not receive allocated intervention

2 Patient preference

2 Unknown reason
2 Lost to follow up
64 Discontinued AGIG
48 Disease progression
7 Received surgical resection
7 Maintenance therapy
2 Physician discretion
1 Obstructive jaundice
1 Femoral head necrosis

64 Analyzed for toxic effects and efficacy 
TABLE 2 | Response rate by treatment group.

\begin{tabular}{|c|c|c|c|c|}
\hline \multirow[t]{2}{*}{ Best Response } & \multirow[t]{2}{*}{ Patients No. $(\%)$ Overall $(n=64)$} & \multicolumn{3}{|c|}{ Patients No. (\%) } \\
\hline & & Head and neck & Body and tail & NA \\
\hline Partial response & $28(43.75)$ & $15(45.45)$ & $10(38.46)$ & $3(6)$ \\
\hline Stable disease & $21(32.81)$ & $10(30.30)$ & $10(38.46)$ & $1(20)$ \\
\hline Progressive disease & $15(23.43)$ & $8(24.24)$ & $6(23.08)$ & $1(20)$ \\
\hline Disease control rate & 49 (76.56) & $25(75.76)$ & $20(76.92)$ & $4(80)$ \\
\hline
\end{tabular}

A Overall Survival

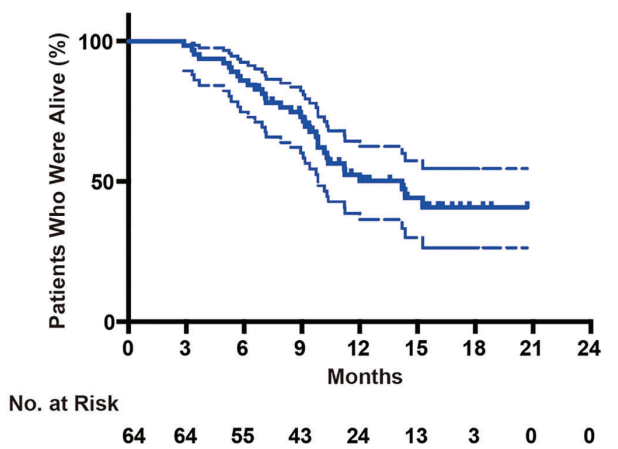

B Progression-free Survival

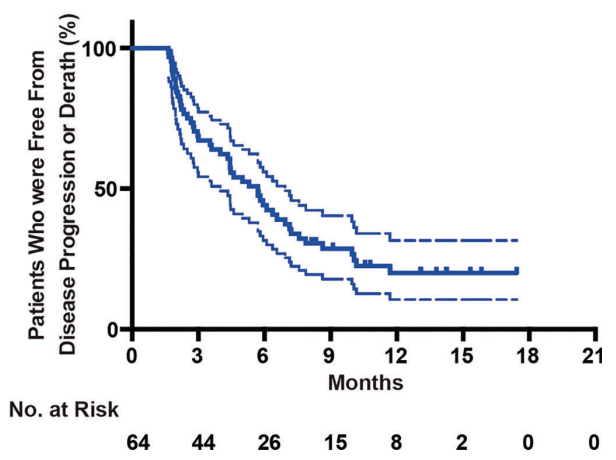

FIGURE 3 | Survival analysis. (A) Overall survival, (B) Progression-free survival.

TABLE 3 | Summary of adverse events.

\begin{tabular}{lcc}
\hline Adverse event & Any grade $(\mathbf{n}=\mathbf{6 4 )}$ & Grade 3-4 (n = 64) \\
\hline White blood cell decreased & $25(39.06 \%)$ & $3(4.69 \%)$ \\
Anemia & $33(51.56 \%)$ & $4(6.25 \%)$ \\
Platelet count decreased & $20(31.25 \%)$ & $5(7.81 \%)$ \\
Neutrophil count decreased & $32(50 \%)$ & $3(4.69 \%)$ \\
Diarrhea & $2(3.13 \%)$ & $0(0 \%)$ \\
Rash maculopapular & $22(34.38 \%)$ & $5(7.81 \%)$ \\
Alopecia & $52(81.25 \%)$ & $32(50 \%)$ \\
Fatigue & $28(43.75 \%)$ & $9(14.06 \%)$ \\
Fever & $48(75 \%)$ & $6(9.38 \%)$ \\
Nausea & $42(65.63 \%)$ & $12(18.75 \%)$ \\
Vomiting & $23(35.94 \%)$ & $15(23.44 \%)$ \\
Dysgeusia & $20(31.25 \%)$ & $0(0 \%)$ \\
Anorexia & $32(50 \%)$ & $8(12.5 \%)$ \\
Peripheral sensory neuropathy & $26(40.6 \%)$ & $10(15.6 \%)$ \\
Adverse event leading to death & $0(0 \%)$ & \\
\hline
\end{tabular}

The effectiveness of the AGIG regimen was also favorable compared to other first-line treatment options presented by previous studies in patients with advanced PC (25-27). Accordingly, we consider the AGIG regimen to be not inferior to the traditional therapy regimen in Chinese patients with advanced PC.

The toxic effects of AGIG were modest. Two patients discontinued chemotherapy early because of obstructive jaundice and femoral head necrosis and none of the patients required discontinuation of AGIG because of toxicity. Exhilaratingly, we observed an obviously decrease in incidences of neutropenia and thrombocytopenia. Totally, only eight patients $(12.5 \%)$ received granulocyte-colony stimulating factor $(3 / 8)$ or thrombopoietin $(5 / 8)$ treatment before or after chemotherapy. We attribute the results to the application of GM-CSF. GM-CSF is an important hematopoietic growth factor and immune modulator. It stimulates the proliferation of macrophage, granulocyte, erythroid, eosinophil, megakaryocyte and multipotent progenitors cells depending on its concentration (28). It also controls eosinophil function in some cases $(29,30)$. Fever was the most common adverse effect in sequential administration period of interleukin-2 and GM-CSF. Rash maculopapular, alopecia, fatigue, nausea, vomiting, peripheral 


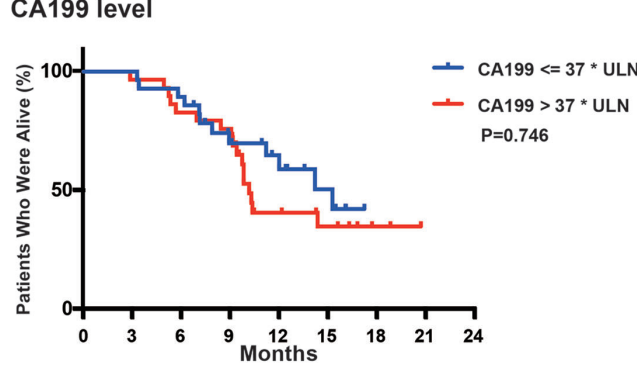

C NK cell count variation

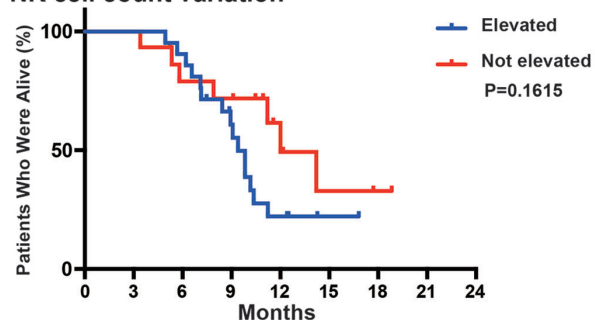

B Eosinophil count variation

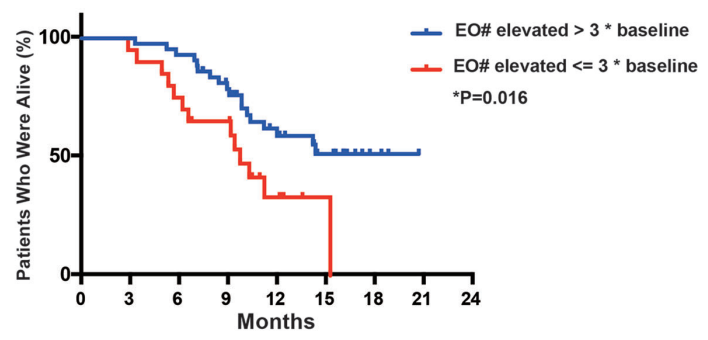

D $\mathrm{CD} 3+\mathrm{CD} 4+/ \mathrm{CD} 3+\mathrm{CD} 8+$ proportion

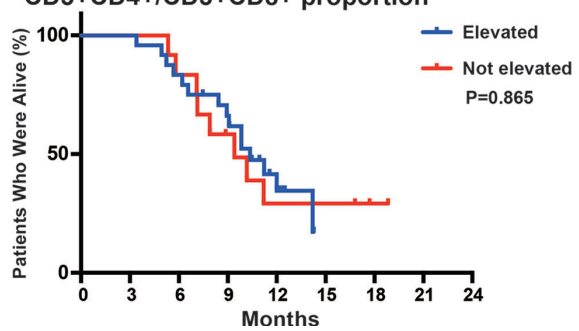

FIGURE 4 | Subgroup analysis in CA199 level (A), Eosinophil count variation (B), NK cell count variation (C) and CD3+CD4/CD3+CD8+ proportion (D). *P $<0.05$.

neuropathy, and neuropsychiatric symptoms were seen with AGIG regimen. However, these effects did not lead to decreased chemotherapy intensity or treatment discontinuation. These findings suggest that lower incidence of myelosuppression in AGIG regimen ensured full dose of drug administration and sufficient course of treatment, which may account for a survival benefit in the trial.

In previous researches, it was explained that the activity of chemo-immunotherapy is mainly depends on the presence of an efficient host's immune response. Cytotoxic drugs were able to induce immunogenic cell death, autophagy and antigen remodeling. In turn, immunological danger signals may empower an efficient tumor-specific immune response $(31,32)$. In subgroup analysis, we found that eosinophil count in the blood elevated three times higher than baseline level predicted a longer survival. But it is a pity that we did not investigate the underlying mechanisms due to the insufficient study design. The increase of eosinophils in cancer patients has been known for over decades (33). To our knowledge, tumor-infiltrating eosinophils was firstly described in human gastric cancers in the 1980s. The infiltrating of eosinophils suggests a good prognostic value for prolonged survival (30). Eosinophils exert anti-tumor effects via direct and indirect mechanisms (34). Eosinophils have been reported to infiltrate multiple tumors, either as an integral part of the tumor microenvironment or in response to various therapeutic strategies. An antitumor role for eosinophils has been demonstrated in various in vitro studies. Eosinophil recruitment, prolonged survival and degranulation have been demonstrated in both human and mouse models (35). The above literatures confirm the phenomenon we observed in this study. Lack of randomization restricts our ability to explore the implicated mechanisms, and further studies are needed.
This study has some limitations. Lack of randomization in a single-arm trial restricts our ability to assess the specific role of AGIG. Insufficient sample size limits the accuracy and authenticity of the results. The improvement of the benefit must be considered hypothesis generating. Given the favorable safety profile and the encouraging antitumor activity of the AGIG regimen, validation by a larger randomized trial is necessary.

In conclusion, the AGIG regimen appears more active and safe than the standard AG chemotherapy. To our knowledge, the study demonstrates the antitumor efficacy of a chemoimmunomodulatory strategy in treating advanced PC sufferers for the first time. These results open a new research area for the treatment of pancreatic cancer by combinatory approaches of cytotoxic chemotherapy and immune modulators. Further investigation is warranted.

\section{CONCLUSION}

The AGIG Chemo-immunotherapy has presented encouraging ORR, DCR, OS, and manageable toxicities as first-line treatment option for advanced PC sufferers. This regimen may be a reliable option for patients with preserved performance status. The improvement of treatment efficiency may result from the activation of non-specific immune response.

\section{DATA AVAILABILITY STATEMENT}

The original contributions presented in the study are included in the article/supplementary material. Further inquiries can be directed to the corresponding authors. 


\section{ETHICS STATEMENT}

The studies involving human participants were reviewed and approved by the Ethics Committee of Drum Tower Hospital. The patients/participants provided their written informed consent to participate in this study. Written informed consent was obtained from the individual(s) for the publication of any potentially identifiable images or data included in this article.

\section{AUTHOR CONTRIBUTIONS}

WD has seen the original study data, reviewed the analysis of the data, approved the final manuscript, and is the author

\section{REFERENCES}

1. Doherty GJ, Tempero M, Corrie PG. HALO-109-301: A Phase III Trial of PEGPH20 (With Gemcitabine and Nab-Paclitaxel) in Hyaluronic Acid-High Stage IV Pancreatic Cancer. Future Oncol (London England) (2018) 14(1):1322. doi: $10.2217 /$ fon-2017-0338

2. Hajatdoost L, Sedaghat K, Walker EJ, Thomas J, Kosari S. Chemotherapy in Pancreatic Cancer: A Systematic Review. Medicina (Kaunas Lithuania) (2018) 54(3):1-7. doi: 10.3390/medicina54030048

3. Hingorani SR, Zheng L, Bullock AJ, Seery TE, Harris WP, Sigal DS, et al. HALO 202: Randomized Phase II Study of PEGPH20 Plus Nab-Paclitaxel/ Gemcitabine Versus Nab-Paclitaxel/Gemcitabine in Patients With Untreated, Metastatic Pancreatic Ductal Adenocarcinoma. J Clin Oncol (2018) 36 (4):359-66. doi: 10.1200/jco.2017.74.9564

4. Giordano G, Pancione M, Olivieri N, Parcesepe P, Velocci M, Di Raimo T, et al. Nano Albumin Bound-Paclitaxel in Pancreatic Cancer: Current Evidences and Future Directions. World J Gastroenterol (2017) 23 (32):5875-86. doi: 10.3748/wjg.v23.i32.5875

5. Goldstein D, El-Maraghi RH, Hammel P, Heinemann V, Kunzmann V, Sastre J, et al. Nab-Paclitaxel Plus Gemcitabine for Metastatic Pancreatic Cancer: Long-Term Survival From a Phase III Trial. J Natl Cancer Inst (2015) 107 (2):1-10. doi: 10.1093/jnci/dju413

6. Burki TK. AZD1775 Plus Chemoradiotherapy for Pancreatic Cancer. Lancet Oncol (2019) 20(9):e472. doi: 10.1016/s1470-2045(19)30537-6

7. Kang J, Hwang I, Yoo C, Kim KP, Jeong JH, Chang HM, et al. Nab-Paclitaxel Plus Gemcitabine Versus FOLFIRINOX as the First-Line Chemotherapy for Patients With Metastatic Pancreatic Cancer: Retrospective Analysis. Invest New Drugs (2018) 36(4):732-41. doi: 10.1007/s10637-018-0598-5

8. Velez-Velez LM, Hughes CL, Kasi PM. Clinical Value of Pharmacogenomic Testing in a Patient Receiving FOLFIRINOX for Pancreatic Adenocarcinoma. Front Pharmacol (2018) 9:1309. doi: 10.3389/fphar.2018.01309

9. Li X, Ma T, Zhang Q, Chen YG, Guo CX, Shen YN, et al. ModifiedFOLFIRINOX in Metastatic Pancreatic Cancer: A Prospective Study in Chinese Population. Cancer Lett (2017) 406:22-6. doi: 10.1016/ j.canlet.2017.07.012

10. Okusaka T, Ikeda M, Fukutomi A, Ioka T, Furuse J, Ohkawa S, et al. Phase II Study of FOLFIRINOX for Chemotherapy-Naïve Japanese Patients With Metastatic Pancreatic Cancer. Cancer Sci (2014) 105(10):1321-6. doi: $10.1111 /$ cas. 12501

11. Zhang DS, Wang DS, Wang ZQ, Wang FH, Luo HY, Qiu MZ, et al. Phase I/II Study of Albumin-Bound Nab-Paclitaxel Plus Gemcitabine Administered to Chinese Patients With Advanced Pancreatic Cancer. Cancer Chemother Pharmacol (2013) 71(4):1065-72. doi: 10.1007/s00280-013-2102-4

12. Von Hoff DD, Ervin T, Arena FP, Chiorean EG, Infante J, Moore M, et al. Increased Survival in Pancreatic Cancer With Nab-Paclitaxel Plus Gemcitabine. N Engl J Med (2013) 369(18):1691-703. doi: 10.1056/NEJMoa1304369

13. Jiang J, Zhou H, Ni C, Hu X, Mou Y, Huang D, et al. Immunotherapy in Pancreatic Cancer: New Hope or Mission Impossible? Cancer Lett (2019) 445:57-64. doi: 10.1016/j.canlet.2018.10.045 responsible for archiving the study files. XQ and CL helped analyzed the data. ZZ, HS, and WK reviewed the analysis of the data and approved the final manuscript. JD and BL helped design the study, analyze the data, and write the manuscript. All authors contributed to the article and approved the submitted version.

\section{FUNDING}

The study was supported by Chen Xiao-Ping Foundation for the Development of Science an Technology of Hubei Province (No. CXPJJH11900001-2019101). The Affiliated Nanjing Drum Tower Hospital of Nanjing University Medical School sponsored this study.

14. Akce M, Zaidi MY, Waller EK, El-Rayes BF, Lesinski GB. The Potential of CAR T Cell Therapy in Pancreatic Cancer. Front Immunol (2018) 9:2166. doi: $10.3389 /$ fimmu.2018.02166

15. De Palma M, Biziato D, Petrova TV. Microenvironmental Regulation of Tumour Angiogenesis. Nat Rev Cancer (2017) 17(8):457-74. doi: 10.1038/ nrc.2017.51

16. Caraglia M, Correale P, Giannicola R, Staropoli N, Botta C, Pastina P, et al. GOLFIG Chemo-Immunotherapy in Metastatic Colorectal Cancer Patients. A Critical Review on a Long-Lasting Follow-Up. Front Oncol (2019) 9:1102. doi: $10.3389 /$ fonc. 2019.01102

17. Correale P, Fioravanti A, Bertoldi I, Montagnani F, Miracco C, Francini G. Occurrence of Autoimmunity in a Long-Term Survivor With Metastatic Colon Carcinoma Treated With a New Chemo-Immunotherapy Regimen. J Chemother (Florence Italy) (2008) 20(2):278-81. doi: 10.1179/joc.2008.20.2.278

18. Dix SP, Gilmore CE. Cytokine Therapy After Bone Marrow Transplantation. Pharmacotherapy (1996) 16(4):593-608. doi: doi:10.1002/j.1875-9114. 1996.tb03641.x

19. Yan WL, Shen KY, Tien CY, Chen YA, Liu SJ. Recent Progress in GM-CSFBased Cancer Immunotherapy. Immunotherapy (2017) 9(4):347-60. doi: 10.2217/imt-2016-0141

20. Koehn TA, Trimble LL, Alderson KL, Erbe AK, McDowell KA, Grzywacz B, et al. Increasing the Clinical Efficacy of NK and Antibody-Mediated Cancer Immunotherapy: Potential Predictors of Successful Clinical Outcome Based on Observations in High-Risk Neuroblastoma. Front Pharmacol (2012) 3:91. doi: 10.3389/fphar.2012.00091

21. Correale P, Botta C, Rotundo MS, Guglielmo A, Conca R, Licchetta A, et al. Gemcitabine, Oxaliplatin, Levofolinate, 5-Fluorouracil, GranulocyteMacrophage Colony-Stimulating Factor, and Interleukin-2 (GOLFIG) Versus FOLFOX Chemotherapy in Metastatic Colorectal Cancer Patients: The GOLFIG-2 Multicentric Open-Label Randomized Phase III Trial. J Immunother (Hagerstown Md 1997) (2014) 37(1):26-35. doi: 10.1097/ cji.0000000000000004

22. Correale P, Tagliaferri P, Fioravanti A, Del Vecchio MT, Remondo C, Montagnani F, et al. Immunity Feedback and Clinical Outcome in Colon Cancer Patients Undergoing Chemoimmunotherapy With Gemcitabine + FOLFOX Followed by Subcutaneous Granulocyte Macrophage ColonyStimulating Factor and Aldesleukin (GOLFIG-1 Trial). Clin Cancer Res (2008) 14(13):4192-9. doi: 10.1158/1078-0432.ccr-07-5278

23. Philip PA, Lacy J, Portales F, Sobrero A, Pazo-Cid R, Manzano Mozo JL, et al. Nab-Paclitaxel Plus Gemcitabine in Patients With Locally Advanced Pancreatic Cancer (LAPACT): A Multicentre, Open-Label Phase 2 Study. Lancet Gastroenterol Hepatol (2020) 5(3):285-94. doi: 10.1016/s2468-1253(19)30327-9

24. Karasic TB, O’Hara MH, Loaiza-Bonilla A, Reiss KA, Teitelbaum UR, Borazanci E, et al. Effect of Gemcitabine and Nab-Paclitaxel With or Without Hydroxychloroquine on Patients With Advanced Pancreatic Cancer: A Phase 2 Randomized Clinical Trial. JAMA Oncol (2019) 5 (7):993-8. doi: 10.1001/jamaoncol.2019.0684

25. Murphy JE, Wo JY, Ryan DP, Clark JW, Jiang W, Yeap BY, et al. Total Neoadjuvant Therapy With FOLFIRINOX in Combination With Losartan 
Followed by Chemoradiotherapy for Locally Advanced Pancreatic Cancer: A Phase 2 Clinical Trial. JAMA Oncol (2019) 5(7):1020-7. doi: 10.1001/ jamaoncol.2019.0892

26. Reni M, Zanon S, Peretti U, Chiaravalli M, Barone D, Pircher C, et al. NabPaclitaxel Plus Gemcitabine With or Without Capecitabine and Cisplatin in Metastatic Pancreatic Adenocarcinoma (PACT-19): A Randomised Phase 2 Trial. Lancet Gastroenterol Hepatol (2018) 3(10):691-7. doi: 10.1016/s24681253(18)30196-1

27. Xu R, Yu X, Hao J, Wang L, Pan H, Han G, et al. Efficacy and Safety of Weekly Nab-Paclitaxel Plus Gemcitabine in Chinese Patients With Metastatic Adenocarcinoma of the Pancreas: A Phase II Study. BMC Cancer (2017) 17 (1):885. doi: 10.1186/s12885-017-3887-z

28. Sugawara Y, Fisher SJ, Zasadny KR, Kison PV, Baker LH, Wahl RL. Preclinical and Clinical Studies of Bone Marrow Uptake of Fluorine-1-Fluorodeoxyglucose With or Without Granulocyte Colony-Stimulating Factor During Chemotherapy. J Clin Oncol (1998) 16(1):173-80. doi: 10.1200/jco.1998.16.1.173

29. Kitamura K. [GM-CSF.G-CSF]. Gan To Kagaku Ryoho Cancer Chemother (1995) 22(2):301-5.

30. Mattei F, Andreone S, Marone G, Gambardella AR, Loffredo S, Varricchi G, et al. Eosinophils in the Tumor Microenvironment. Adv Exp Med Biol (2020) 1273:1-28. doi: 10.1007/978-3-030-49270-0_1

31. Williams P, Galipeau J. GMCSF-Interleukin Fusion Cytokines Induce Novel Immune Effectors That Can Serve as Biopharmaceuticals for Treatment of Autoimmunity and Cancer. J Internal Med (2011) 269(1):74-84. doi: 10.1111/ j.1365-2796.2010.02314.x

32. Kabacaoglu D, Ciecielski KJ, Ruess DA, Algül H. Immune Checkpoint Inhibition for Pancreatic Ductal Adenocarcinoma: Current Limitations and Future Options. Front Immunol (2018) 9:1878. doi: 10.3389/fimmu.2018.01878
33. Chusid MJ. Eosinophils: Friends or Foes? J Allergy Clin Immunol Pract (2018) 6(5):1439-44. doi: 10.1016/j.jaip.2018.04.031

34. Dennis KL, Wang Y, Blatner NR, Wang S, Saadalla A, Trudeau E, et al. Adenomatous Polyps are Driven by Microbe-Instigated Focal Inflammation and Are Controlled by IL-10-Producing T Cells. Cancer Res (2013) 73 (19):5905-13. doi: 10.1158/0008-5472.can-13-1511

35. Grisaru-Tal S, Itan M, Klion AD, Munitz A. A New Dawn for Eosinophils in the Tumour Microenvironment. Nat Rev Cancer (2020) 20(10):594-607. doi: 10.1038/s41568-020-0283-9

Conflict of Interest: The authors declare that the research was conducted in the absence of any commercial or financial relationships that could be construed as a potential conflict of interest.

Publisher's Note: All claims expressed in this article are solely those of the authors and do not necessarily represent those of their affiliated organizations, or those of the publisher, the editors and the reviewers. Any product that may be evaluated in this article, or claim that may be made by its manufacturer, is not guaranteed or endorsed by the publisher.

Copyright (C) 2021 Dai, Qiu, Lu, Zou, Sha, Kong, Liu and Du. This is an open-access article distributed under the terms of the Creative Commons Attribution License (CC BY). The use, distribution or reproduction in other forums is permitted, provided the original author(s) and the copyright owner(s) are credited and that the original publication in this journal is cited, in accordance with accepted academic practice. No use, distribution or reproduction is permitted which does not comply with these terms. 